\title{
FDA Approves Eteplirsen for Duchenne Muscular Dystrophy: The Next Chapter in the Eteplirsen Saga
}

\author{
Annemieke Aartsma-Rus ${ }^{1}$ and Arthur M. Krieg ${ }^{2}$
}

Keywords: regulatory, oligonucleotide, splicing modulation, therapy

$\mathbf{E}$ TEPLIRSEN, A PHOSPHORODIAMIDATE morpholino antisense oligonucleotide (PMO) that modulates splicing to treat Duchenne muscular dystrophy (DMD) patients, received accelerated approval by Food and Drug Administration (FDA) on September 19, 2016 [1]. This heralded a number of firsts: it is the first drug that is approved for DMD in the United States, the first approved oligonucleotide that modulates splicing, the first approved PMO, and also the first oligonucleotide to be approved based on very limited data. Although its approval was shrouded in controversy, eteplirsen could also become the first oligonucleotide to be a commercial success. This brief commentary reviews the disease, the therapy, the clinical data, and what eteplirsen's approval means for the oligonucleotide therapeutics field.

DMD is an X-linked progressive disease by which patients lose muscle function from an early age, resulting in wheelchair dependency generally around the age of 12 , the need for assisted ventilation around the age of 20 , and premature death in the third-fourth decade of life. Apart from symptomatic care, there is no treatment; while their peers gain skills and become independent, DMD patients will lose one function after another until they rely on around-the-clock care before they die prematurely. Given the severe and progressive nature and the unmet medical need, the disease clearly qualifies for the FDA-accelerated approval pathway.

DMD is caused by mutations in the dystrophin gene. Normally, dystrophin stabilizes muscle fibers during contraction by connecting the actin cytoskeleton within muscle fibers to the extracellular matrix surrounding muscle fibers. DMD patients have mutations that disrupt the reading frame and/or cause premature truncation of protein translation. Interestingly, mutations that maintain the reading frame allow the production of an internally deleted protein that is partially functional, owing to the fact that the crucial domains of dystrophin are located at the very beginning and end of the protein, whereas the middle is largely redundant. These internally deleted dystrophins are found in the later onset and less progressive Becker muscular dystrophy.

The exon skipping approach stems from the fact that most DMD patients have in theory the genetic capacity to produce Becker-like dystrophins. Using antisense oligonucleotides (AONs) to interfere with the splicing process, the reading frame can be restored to allow the production of a partially functional Becker-type dystrophin rather than a nonfunctional DMD-type dystrophin. Depending on the mutation present in any individual patient, one or more different exons may need to be skipped to restore the reading frame. However, because mutations cluster, skipping certain exons is expected to be therapeutic in larger groups of patients, with most notably exon 51 skipping applying to $13 \%-14 \%$ of patients. Ultimately, exon skipping of various exons may be an effective therapy in more DMD patients.

After obtaining proof of concept in patient-derived cell models and animal models, two AON chemistries were clinically developed for exon skipping in DMD. The 2'-O-methyl phosphorothioate exon 51 skipper drisapersen was developed by Prosensa/GSK/BioMarin. After a first dose-finding study, 12 DMD patients were treated on and off with $6 \mathrm{mg} / \mathrm{kg}$ for more than 6 years in an open-label study. Eight of the 10 ambulant patients were stable in the distance walked in $6 \mathrm{~min}$ for the duration of the study, whereas 2 patients lost ambulation [2]. Drisapersen was then tested in two phase 2 and one phase 3 placebo-controlled trials in more than 300 DMD patients. The primary endpoint in these trials was the 6-min walk test. Although there was a tendency of drisapersen-treated patients to walk further than placebo-treated patients, the difference was small and not statistically or clinically significant.

Post hoc analysis suggested that treatment may have had a therapeutic effect on younger patients and a marketing approval application was filed. FDA did not grant this, based on

\footnotetext{
${ }^{1}$ Leiden University Medical Center, Leiden, the Netherlands.

${ }^{2}$ Checkmate Pharmaceuticals, Cambridge, Massachusetts.
}

(C) Annemieke Aartsma-Rus, et al., 2017; Published by Mary Ann Liebert, Inc. This Open Access article is distributed under the terms of the Creative Commons Attribution Noncommercial License (http://creativecommons.org/licenses/by-nc/4.0/) which permits any noncommercial use, distribution, and reproduction in any medium, provided the original author(s) and the source are credited. 
the facts that the pivotal trials failed to demonstrate significant clinical benefit (or clear increase in dystrophin expression) and that significant toxicities were observed (skin reactions and proteinuria in many patients and severe thrombocytopenia in about $2 \%$ of patients). After the FDA rejection of drisapersen's application, BioMarin recently withdrew its marketing authorization application with the European Medicine Agency and announced its decision to stop clinical development of its exon skipping compounds and to focus on the next generation of compounds [3].

The PMO exon 51 skipper eteplirsen has been tested in a far smaller cohort of patients. A first dose-finding study revealed that the highest dose tested, $20 \mathrm{mg} / \mathrm{kg}$, was suboptimal. Then a new study was initiated in 2011 in 12 patients, comparing 30, $50 \mathrm{mg} / \mathrm{kg}$, and placebo weekly for 24 weeks, after which the placebo group was switched to 30 or $50 \mathrm{mg} / \mathrm{kg}$ dosing. All 12 patients have now been treated weekly for more than 4 years without apparent treatment-related side effects.

The primary endpoint was dystrophin restoration as assessed by the percentage of muscle fibers that express detectable dystrophin by immunohistochemistry of muscle biopsies. In biopsies taken at baseline, 12, 24, and 48 weeks, the number of dystrophin-positive fibers was counted subjectively in a blinded manner and was initially reported to be significantly increased to $30 \%-50 \%$ of dystrophin-positive fibers for the 24- and 48-week-treated specimens [4]. However, subsequent reanalysis of these biopsy slides performed by the FDA raised questions regarding the magnitude of any increase in dystrophin expression [5].

To more rigorously evaluate the possible restoration of dystrophin expression, FDA requested dystrophin amounts to be quantified by a well-controlled western blot assay, and this was performed on additional biopsies taken after 188 weeks of treatment. This revealed average levels of $0.93 \%$ of the normal amount of dystrophin, compared with a baseline value of $0.08 \%$ in biopsies of untreated Duchenne muscles. This suggested that the $30 \%-50 \%$ fibers designated as positive each produce only small amounts of dystrophin. Indeed, a rigorously controlled assessment of the percentage of muscle fibers expressing detectable dystrophin by immunohistochemistry showed an increase from $1.1 \%$ of dystrophin-expressing muscle fibers in untreated samples to $17.4 \%$ of the muscle fibers in treated biopsies, and the intensity of dystrophin expression in positive fibers increased from $9.4 \%$ of normal at baseline to $22.6 \%$ of normal in the treated biopsies [6].

Patients were assessed for their functional status primarily with the 6-min walk test, but lacking a placebo group beyond 24 weeks of treatment, and having two treated patients who lost ambulation within 3 months of trial initiation, the only comparison possible was with historical controls selected from natural history data of baseline-matched patients from Belgium and Italy [7]. Using historical controls as a comparison, a slower decrease in the distance walked in $6 \mathrm{~min}$ in eteplirsen-treated patients was observed than would be expected from natural history. However, such comparisons in small groups of patients are challenging to interpret, and the FDA reviewers were not convinced that the 6-min walk times demonstrated any benefit.

Reluctant to approve eteplirsen based on such a small number of patient samples, the FDA asked Sarepta in June 2016 to provide evidence of increased dystrophin expression by western blot from additional biopsies of eteplirsen-treated patients in a new ongoing clinical trial before they would make a decision. Sarepta had initiated a confirmatory phase 3 trial with eteplirsen (PROMOVI) in September 2014 [8]. In this study, 80 ambulant DMD patients whose dystrophin mutations were amenable to exon 51 skipping have been receiving weekly dosing of eteplirsen at $30 \mathrm{mg} / \mathrm{kg}$, while 80 matched DMD patients with mutations not amenable to exon 51 skipping do not receive treatment, but are followed with the same functional outcome measures.

Thirteen patients in the PROMOVI study had been treated for at least 48 weeks with eteplirsen, and western blot analysis of biopsies taken from these patients before and after 48 weeks of treatment revealed an increase from $0.16 \%$ at baseline to $0.44 \%$ at 48 weeks on average, with the caveat that not all patients demonstrated a post-treatment response in dystrophin on Sarepta's validated western blot system $[9,10]$. In fact, about half of the patients had no or minimal apparent increase in dystrophin expression and the increases in the others were higher, up to more than $1 \%$ of normal [10]. The lower increases in dystrophin expression at 48 weeks compared with the 180-week biopsies may be related to the longer duration of therapy.

Although the levels of dystrophin restoration are disappointing, the increases of $0.28 \%$ and $0.93 \%$ do provide evidence for the intended mechanism of action for eteplirsen: the FDA reviewers agreed that eteplirsen increases dystrophin levels in some patients. What the data do not tell us is whether this very small amount of dystrophin expression will be enough to slow down disease progression. Such modest increases in dystrophin may seem negligible, and some FDA reviewers of the eteplirsen application wanted to reject it because of their beliefs that such small increases are not going to provide any therapeutic benefit [10].

Nevertheless, earlier preclinical studies in mouse models have revealed that a little bit of dystrophin may go a long way, with $<4 \%$ dystrophin significantly increasing survival in a severe background. Furthermore, it has become clear that patients requiring exon 44 skipping have higher baseline levels of dystrophin, and also have a slower disease progression than patients with other mutations. The dystrophin levels in these patients, however, have not been quantified with a validated western blot system, so how they relate to the increases found after eteplirsen treatment is unclear. Furthermore, these mice and patients have had these low amounts of dystrophin since birth. The question that one cannot answer yet is whether an increase to $0.28 \%-0.93 \%$ of dystrophin can be sufficient to slow down disease progression once pathology is established.

FDA indeed stated in their approval that "A clinical benefit of Exondys 51 (tradename of eterplirsen), including improved motor function, has not been established" [1] and mandated that Sarepta will have to perform confirmatory studies to establish that eteplirsen can slow down disease progression. Within the FDA, there was intense and unprecedented disagreement concerning eteplirsen's approval, which was made public on September 16, 2016, when the FDA commissioner released a document summarizing in remarkable detail the internal controversies surrounding the eteplirsen review [10]. In brief, the Director of the Office of Drug Evaluation, Dr. Ellis Unger, and the Division of Neurology Products, headed by Dr. Ronald Farkas, had initially rejected the eteplirsen application. However, they were overruled by Dr. Janet Woodcock, the Director of CDER. An internal FDA dispute resolution process was then initiated, culminating in 
an appeal to the FDA Commissioner, Dr. Robert Califf, who allowed Dr. Woodcock's approval decision to stand [10]. Dr. Farkas and at least one other member of the eteplirsen review team resigned from the FDA. Although the reason for these resignations has not been made public, the timing led to speculation that they are related to the eteplirsen approval. Regardless of the controversies, the patient community celebrates the approval as a victory, pointing to the fact that 10 of the 12 boys in the original trial are still walking and functioning at a level higher than expected in DMD. Indeed, a group of 36 prominent DMD clinician-scientists had published a letter calling for eteplirsen's approval based on the dystrophin data and clinical results [11]. Meanwhile, some critical voices argue that eteplirsen is a very expensive placebo. Several insurance companies have already approved coverage of eteplirsen and patients have begun receiving it, but one major insurance company, Anthem, indicated on October 7, 2016, that they will not reimburse eteplirsen treatment [12]. Eteplirsen treatment will cost in the order of $\$ 300,000-400,000$ per year per patient initially, meaning that without reimbursement, most patients in the United States will not have access to the drug.

What lessons does eteplirsen's approval hold for the oligonucleotide therapeutics community? One point worth noting is that, like other drugs, oligonucleotides are going to be reviewed and approved or rejected based on their merits. Some commentators on the eteplirsen controversy have expressed fears that approval of a drug based on such a small data set means that the FDA has "lowered the bar" and that more companies will now seek approval of marginally effective drugs based on small, underpowered clinical trials and unvalidated endpoints. That would be a mistake, there is no indication that FDA will "lower the bar" for other rare disease drugs and/or oligonucleotides that are currently in development. One should also not forget that the road to approval for eteplirsen was long, with plans for a new drug application announced already in 2013 [13].

If there is one lesson from the eteplirsen saga for drug developers, it is that clinical development always should be rigorous and well controlled, and that working with the FDA in a constructive manner is the best way to rapidly deliver new therapeutics for patients in need, perhaps using the ongoing dialogue between the DMD patient community, academics, and regulators in Europe as an example [14].

In all this controversy, one can only hope that eteplirsen indeed does work and that longer term treatment will reveal a slower disease progression in DMD patients. Eteplirsen may have only a modest effect on restoring dystrophin expression, but there is reason to expect that even small amounts of dystrophin could provide clinical benefits to patients. DMD patients and parents face the consequences of this disease on a daily basis, coping with progressive loss of function and dreading loss of the next function. These patients deserve an effective drug and hope for more effective drugs than eteplirsen to be developed in the future.

\section{Author Disclosure Statement}

A.A.R. report being employed by LUMC, which has patents on exon skipping technology. As a coinventor of some of these patents, A.A.R. may receive a share of potential roy- alties. A.A.R. reports being an ad hoc consultant for BioMarin, PTC, BMS, and Summit. Remuneration for these activities and speaker honoraria for BioMarin and PTCorganized satellite symposia go to LUMC. A.M.K. was previously employed as Sarepta's CSO and has been a consultant to companies working on RNA and DMD therapeutics.

\section{References}

1. www.fda.gov/NewsEvents/Newsroom/PressAnnouncements/ ucm521263.htm

2. Goemans NM, M Tulinius, $M$ van den Hauwe, AK Kroksmark, G Buyse, RJ Wilson, JC van Deutekom, SJ de Kimpe, A Lourbakos, and Campion G. (2016). Long-term efficacy, safety, and pharmacokinetics of drisapersen in Duchenne muscular dystrophy: results from an open-label extension study. PLoS One 11:e0161955.

3. http://investors.bmrn.com/releasedetail.cfm?ReleaseID= 973536

4. Mendell JR, LR Rodino-Klapac, Z Sahenk, K Roush, L Bird, LP Lowes, L Alfano, AM Gomez, S Lewis, et al; Eteplirsen Study Group. (2013). Eteplirsen for the treatment of Duchenne muscular dystrophy. Ann Neurol 74:637-647.

5. www.fda.gov/downloads/advisorycommittees/committees meetingmaterials/drugs/peripheralandcentralnervoussystem drugsadvisorycommittee/ucm481912.pdf

6. www.fda.gov/downloads/advisorycommittees/committees meetingmaterials/drugs/peripheralandcentralnervoussystem drugsadvisorycommittee/ucm497064.pdf

7. Mendell JR, N Goemans, LP Lowes, LN Alfano, K Berry, J Shao, EM Kaye, and E Mercuri; Eteplirsen Study Group and Telethon Foundation DMD Italian Network. (2016). Longitudinal effect of eteplirsen versus historical control on ambulation in Duchenne muscular dystrophy. Ann Neurol 79: 257-271.

8. https://clinicaltrials.gov/ct2/show/NCT02255552

9. Presented by Prof. Francesco Muntoni at the Sarepta sponsored satellite symposium of the World Muscle Society, October 2016, Granada, Spain

10. www.accessdata.fda.gov/drugsatfda_docs/nda/2016/206488_ summary\%20review_Redacted.pdf

11. www.cdmd.ucla.edu/files/view/FDA_ETEPLIRSEN_LET TER_02242016.pdf

12. www.srnnews.com/anthem-says-will-not-cover-first-fdaapproved-duchenne-drug

13. www.drugs.com/nda/eteplirsen_130725.html

14. Straub V, P Balabanov, K Bushby, M Ensini, N Goemans, A De Luca, A Pereda, R Hemmings, G Campion, et al. (2016). Stakeholder cooperation to overcome challenges in orphan medicine development: the example of Duchenne muscular dystrophy. Lancet Neurol 15:882-890.

Address correspondence to: Annemieke Aartsma-Rus, PhD Leiden University Medical Center Postzone S4-P, Albinusdreef 2 2333 ZA Leiden The Netherlands

E-mail: a.m.aartsma-rus@lumc.nl

Received for publication October 26, 2016; accepted after revision November 7, 2016. 